\title{
Efficient Application of Selected Mapping with Clipping for PAPR Reduction in OFDM Systems
}

\author{
Anusha Chacko and J. Jeevitha
}

\begin{abstract}
Orthogonal Frequency Division Multiplexing (OFDM) is an inimitable technology in wireless communication. High peak-to-average power ratio (PAPR) is a challenging issue in OFDM system. Large PAPR results in significant distortion in OFDM signal when passed through nonlinear high power amplifier. Selected mapping (SLM) is an efficient technique to reduce PAPR. In this work we efficiently apply SLM technique with clipping to reduce PAPR. And the simulation results show that the proposed techniques have better PAPR reduction performance than conventional Selected Mapping technique and SLM with interleaving.
\end{abstract}

Index Terms--- OFDM, PAPR, SLM, CCDF, IFFT, HPA, ISI

\section{INTRODUCTION}

$\mathrm{H}$ IGH data rate is one of the most desirable qualities in a communication system. But in communication systems with single carrier modulation, symbol duration reduces with increase in data rate. This leads to Inter Symbol Interference (ISI). The issue of ISI can be reduced with Orthogonal Frequency Division Multiplexing. OFDM is a promising technology in wireless communication [1]. The basic idea in OFDM is the use of narrow band subcarriers. Instead of using a single wide band subcarrier OFDM uses multiple narrow band subcarriers for data transmission.

Orthogonal Frequency Division multiplexing is a multicarrier modulation scheme. And each subcarrier is orthogonal to each other. OFDM is utilized in various standards like IEEE 802.11a, Digital Audio Broadcasting and digital Video Broadcasting. IEEE 802.11a adopts OFDM with 52 subcarriers.

High Peak-to-Average Power Ratio is the major issue in Orthogonal Frequency Division Multiplexing [2, 11]. OFDM signal has wide amplitude range. This is the reason behind the high Peak-to-Average Power Ratio. PAPR can be defined as the peak amplitude squared divided by mean value squared.

The nonlinear high power amplifiers have high power efficiency than linear high power amplifiers. But in the case of

Anusha Chacko, Department of Electronics and Communication, Karunya University, Coimbatore, India.E-mail: anushastella@gmail.com

J. Jeevitha, Department of Electronics and Communication, Karunya University, Coimbatore, India. E-mail: jeevithajoseph@karunya.edu

\section{DOI: 10.9756/BIJRCE.4872}

OFDM systems we cannot go for nonlinear high power amplifier. The nonlinear HPA causes adjacent channel interference and in-band distortion [13]. The high PAPR demands linear high power amplifier with wide range.

But these are of high implementation cost. And linear high power amplifiers have low efficiency in amplification. The efficiency of an amplifier is very important in communication systems, because it decides even the range of communication. The only solution to these problems is the reduction of Peak-to Average Power Ratio in OFDM signal.

There are many PAPR reduction techniques proposed. In the PAPR reduction techniques the signal scrambling techniques introduces no distortion to the data to be transmitted. Partial Transmit Sequence, Selected Mapping, Clipping [5, 10, 12], Coding and Non-linear companding transforms [9] are the well-known PAPR reduction techniques in OFDM system [3].

Among these Clipping is the simplest technique to reduce high Peak-to-Average Power Ratio in OFDM systems. In Clipping amplitude of the signal above a certain threshold is clipped. But this results in in-band distortion and out of band radiation [2]. Filtering is commonly used along with clipping to avoid out of band radiation.

Non linear Companding Technique is similar to clipping. In clipping technique the amplitude of the signal above a particular threshold is clipped, where as in Nonlinear Companding Techniques, the companding transforms compand the OFDM signals. And at the receiver side the inverse companding transforms can be used to recover the original OFDM signal [3].

The major reason of high Peak-to-Average Power Ratio in OFDM is due to addition of number of sub carriers. And when the signals with same phase add together high peaks occurs. The coding technique minimizes the occurence of the probability of the same phase signal.

Partial Transmit Sequence and Selected Mapping are the commonly used signal scrambling techniques. The Partial Transmit Sequence technique introduces no distortion to the data transmitted.

In PTS the data block is divided into sub blocks [4]. Then Inverse Fast Fourier Transform is applied to these sequences. Thus the sequences are converted into time domain. These partial transmit sequences are then multiplied with generated phase factors [6]. Then the sub blocks are optimally combined to form the signal with minimum PAPR.

Selected Mapping is an efficient technique to reduce peakto-average power ratio in Orthogonal Frequency Division 
Multiplexing systems. In SLM the data block is multiplied with many phase sequences. And from the signals the signal with minimum PAPR is selected for transmission.

Complimentary Cumulative Distribution Function (CCDF) of Peak-to-Average Power Ratio is used to show the PAPR reduction performance of the techniques.

\section{SELECTED MAPPING}

Selected Mapping is a signal scrambling technique. SLM introduces no distortion to the data. In Selected Mapping the data block is multiplied by number phase sequences generated [7]. The phase sequence is a set of values with magnitude unity and phase randomly selected.

The key concept in SLM is the generation of phase sequences. The same data block is multiplied with all the phase sequences generated [8]. This results in number of signals which carry same information [14]. Then Inverse Fast Fourier Transform is applied for each signal generated. And from these alternative signals the signal with minimum Peakto-Average Power Ratio is selected for transmission [15].

Figure 1 shows the block diagram of Selected Mapping technique. Here the phase sequences Q1, Q2 .......QL are generated. And $\mathrm{D}$ represents the data block. Then the data $\mathrm{D}$ is multiplied with each of the phase sequences Q1, Q2 .....QL. And Inverse fast Fourier Transform is applied to each of the signals. From these signals the signal with minimum peak-toaverage power ratio is selected for transmission.

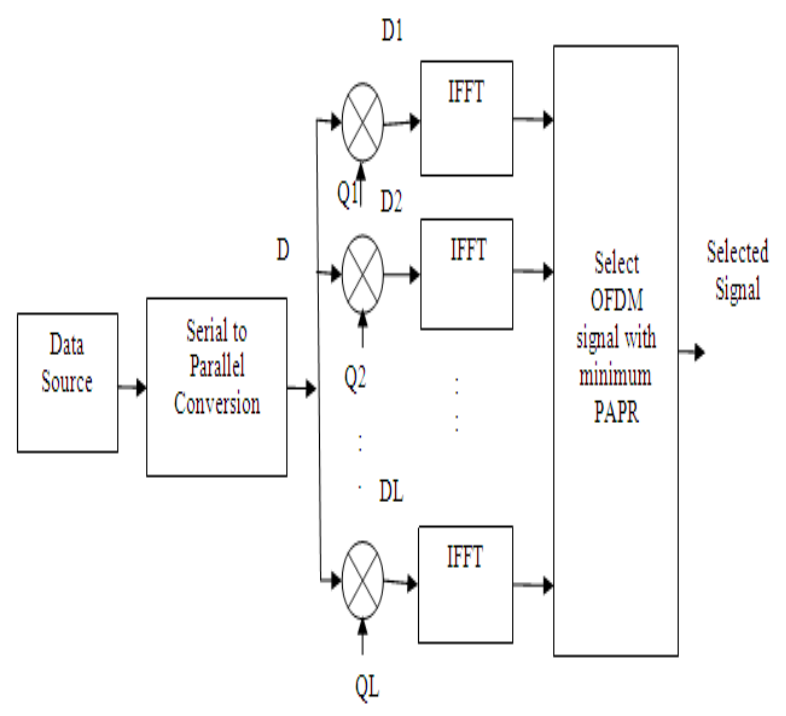

Fig. 1: Selected Mapping Technique

\section{EFFICIENT APPLICATION OF SELECTED MAPPING}

In the proposed techniques SLM is applied efficiently for PAPR reduction in OFDM systems. Here the input data is divided into two sub blocks. And then SLM is applied to each of the sub blocks separately. The technique gives better PAPR reduction than the conventional SLM.

Segmentation and Interleaving [16] are adopted to divide the input data block into two sub blocks. Figure 2 shows the block diagram of efficient application of Selected Mapping technique. The block diagram is same for both segmentation based technique and interleaving based technique.

The only difference between the two techniques is the method adopted to divide the data block into two sub blocks. In segmentation based technique, segmentation is used to divide the data block into two sub blocks. And in interleaving based technique interleaving is used to divide the input data block into two sub blocks.

Figure 2 shows the block diagram of proposed method. Here the input data block is divided into two sub blocks. And then Selected Mapping is applied to each of the two sub blocks separately.

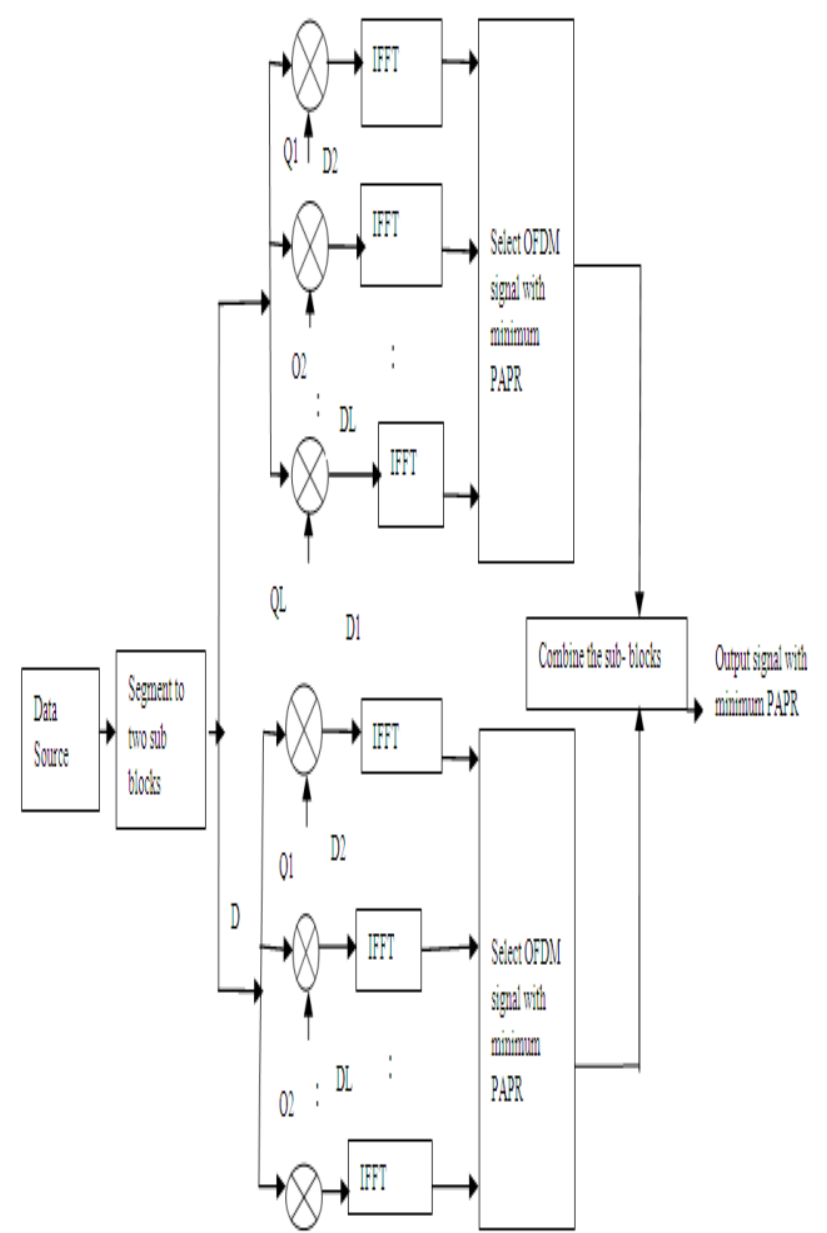

Fig. 2: Efficient Application of Selected Mapping Technique

Finally the two sub blocks are combined to form the output signal. And the techniques resulted in better PAPR reduction performance than conventional Selected Mapping.

\section{A. Segmentation}

In this technique the input data block is divided into first half section and second half section. And Selected Mapping is applied to each half section separately. The two sub sections are then combined to obtain the final output signal.

\section{B. Interleaving}

In interleaving the input data block is divided into even indexed and odd indexed. And then Selected Mapping 
technique is applied to each the sub blocks separately. Finally the two sub blocks are combined together for the final output signal.

\section{EFFICIENT APPLICATION OF SLM with CLIPPING}

In efficient application of selected mapping with clipping, clipping is applied before the efficient application of selected mapping. To divide the input data block into two sub blocks interleaving is used. Here first clipping is applied to the input data. Then the data block is divided into even indexed data block and odd indexed data block. After that selected mapping technique is applied separately to each of the sub blocks. Finally the blocks are combined together to get the signal with minimum PAPR.

\section{RESULTS}

The results are simulated in MATLAB. And to represent the PAPR reduction performance Complimentary Cumulative Distribution Function (CCDF) of PAPR is used. The simulation results showed that the proposed techniques have better PAPR reduction capability than conventional Selected Mapping technique.

The $\mathrm{x}$-axis of CCDF plot of PAPR represents the threshold values and the $y$-axis shows the probability that the PAPR is greater than that threshold. Figure 3 shows the CCDF plot of OFDM signal with no reduction techniques applied. For simulation of the results OFDM with 64 subcarriers is used. And this shows original Orthogonal Frequency Division Multiplexing signals have high Peak-to-Average Power Ratio.

Figure 4 shows the PAPR reduction performance of the conventional Selected Mapping technique. And the graph shows that SLM is an efficient PAPR reduction technique. Selected Mapping can achieve good PAPR reduction without any distortion to the original signal.

The figure 5 shows the proposed techniques. The maximum threshold value with probability of occurrence of OFDM signal is reduced than the conventional Selected Mapping technique. And this proves, the proposed techniques have better PAPR reduction performance than the conventional Selected Mapping technique. The results showed that the Selected Mapping with interleaving has better PAPR reduction performance than the Selected Mapping with segmentation.

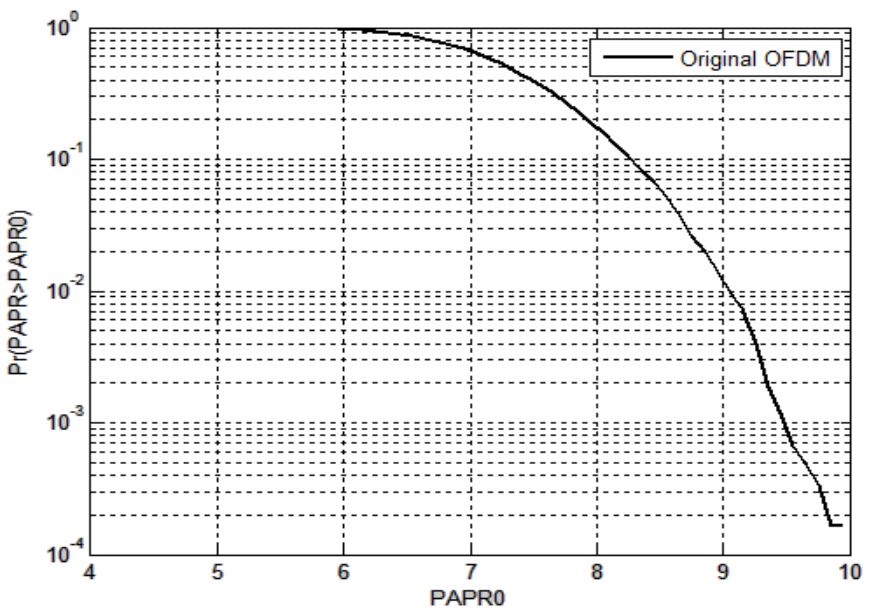

Fig. 3: CCDF Plot of PAPR for the Original OFDM Signal

Figure 3 shows the high Peak-to-Average Power Ratio of OFDM system. In the plot there is probability of PAPR greater than the threshold value exists even near a threshold of $10 \mathrm{~dB}$, which implies PAPR of OFDM is very high.

Figure 4 shows the CCDF plot of PAPR for Selected Mapping technique and original OFDM signal. Original OFDM signal has PAPR up to $10 \mathrm{~dB}$. And the Selected Mapping technique limited the maximum PAPR below $7.5 \mathrm{~dB}$.

Figure 5 shows the CCDF plot of PAPR for the proposed techniques. The proposed techniques limited the maximum PAPR value below $6.5 \mathrm{~dB}$. And from the graph it is clear that the SLM with interleaving technique has slightly higher PAPR reduction performance than the SLM with Segmentation technique.

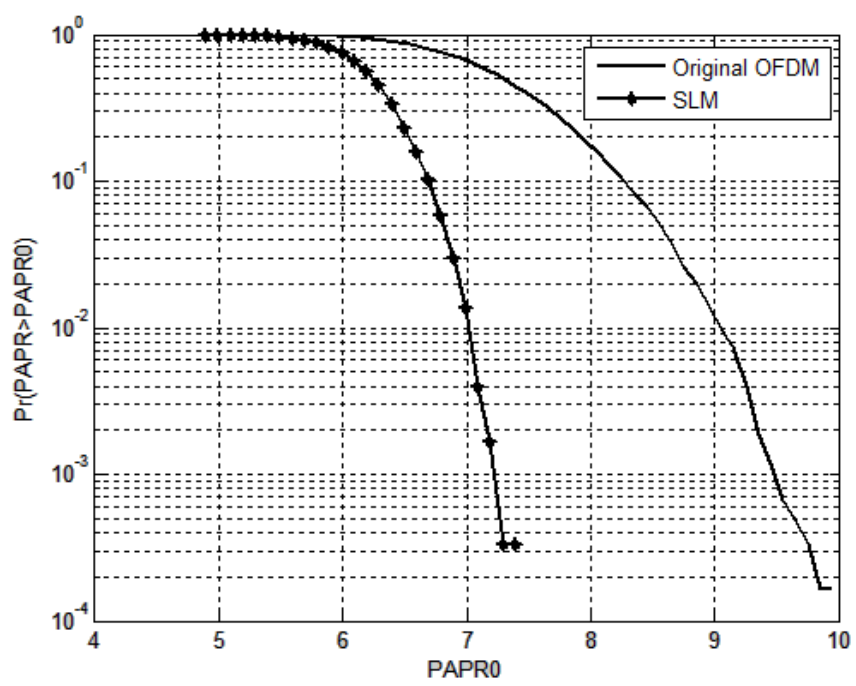


Fig. 4: CCDF plot of PAPR for the Original OFDM Signal and OFDM Signal with SLM

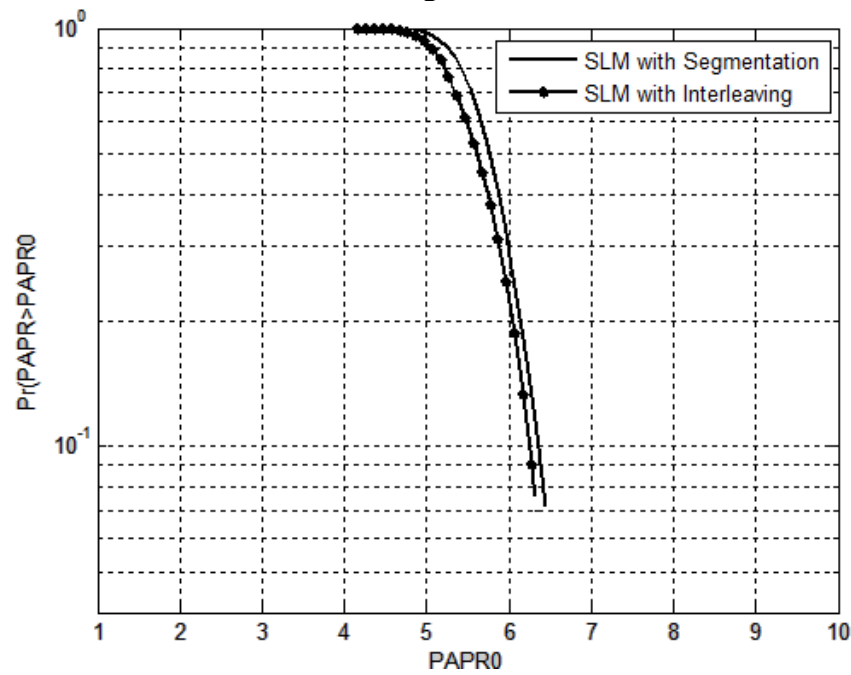

Fig. 5: CCDF Plot of PAPR for SLM with Segmentation and SLM with Interleaving

Figure 6 shows the CCDF plot of PAPR for efficient application of SLM with clipping. From the simulation results we can see that SLM with interleaving has better PAPR reduction than SLM with segmentation. So clipping is added with SLM with interleaving. And the simulation results shows that efficient application of selected mapping with clipping achieves better result than SLM with interleaving. In clipping the part of signal above a particular threshold is clipped. In figure 6 the maximum threshold is reduced up to $4.9 \mathrm{~dB}$. The maximum threshold value represents the maximum PAPR value present after the application of a particular PAPR reduction technique.

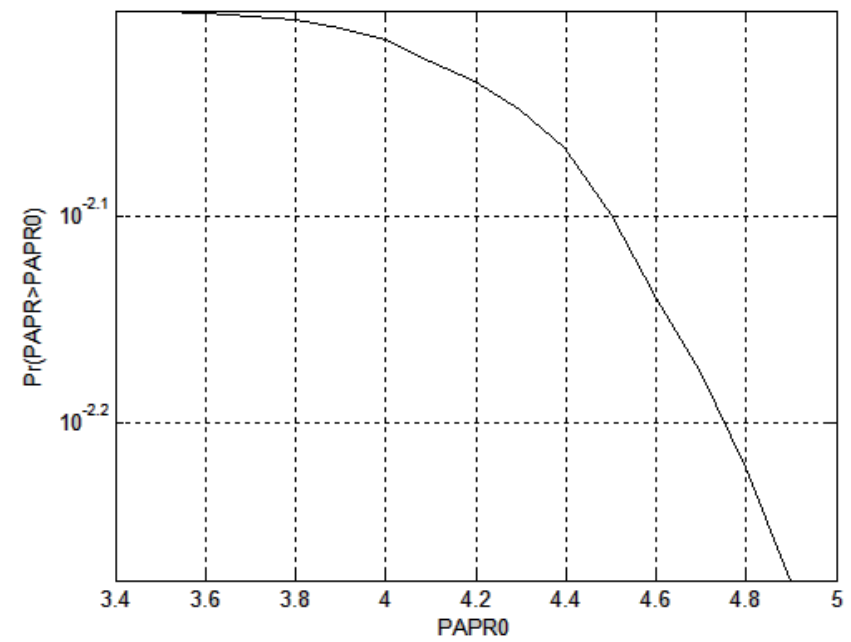

Fig. 6: CCDF Plot of PAPR for Efficient Application of SLM with Clipping

\section{CONCLUSION}

OFDM is a promising technology in wireless communication. And high PAPR is the major challenging issue in OFDM systems. Selected Mapping is an efficient method to limit high PAPR. In this work we have proposed SLM with Segmentation and SLM with Interleaving. And the techniques resulted in further reduction in PAPR than the conventional
Selected Mapping. Among the proposed techniques SLM with Interleaving has better PAPR reduction performance.

\section{REFERENCES}

[1] Byung Moo Lee, Youngok Kim, and Rui J.P. de Figueiredo, "Performance Analysis of The Clipping Scheme with SLM Technique for PAPR Reduction of OFDM Signals in Fading Channels" Wireless Pers. Commun., pp. 331-344, September 2010.

[2] Seung Hee Han, Jae Hong Lee "An Overview of Peak-to-Average Power Ratio Reduction Techniques for Multicarrier Transmission", IEEE Wireless Communications, pp. 56-65, April 2005.

[3] Tao Jiang, Yiyan Wu, "An Overview: Peak-to-Average Power Ratio Reduction Techniques for OFDM signals", IEEE Transactions on Broadcasting, VOL.54, pp. 257-268, June 2008.

[4] Jing gao, Jinkuan Wang, Xin Song, and Bin Wang, "PAPR Reduction With Low Computational Complexity For OFDM Systems", Physics Procedia, pp. 1401 - 1407, May 2012.

[5] Byung Moo Lee, Youngok Kim, "An Adaptive Clipping And Filtering Technique for PAPR Reduction of OFDM Signals" Circuits Syst. Signal Process., pp. 1335-1349, November 2012.

[6] Jun Hou, JianhuaGe, and Jing Li (2011) "Peak-To-Average Power Ratio Reduction of OFDM Signals Using PTS Scheme With Low Computational Complexity" IEEE Transactions On Broadcasting, Vol. 57, pp. 143-148, March 2011

[7] L. Yang, Y. M. Siu, K.K. Soo, S.W Leung and S.Q Li, "LowComplexity PAPR Reduction Technique For OFDM Systems Using Modified Widely Linear SLM Scheme" Int. J. Electron. Commun., pp. 1006- 1010, May 2012.

[8] Saber Meymanatabadi, Javad Musevi Niya, Behzad Mozaffari, "Selected Mapping Technique for PAPR Reduction without Side Information Based on m-Sequence" Wireless Pers. Commun., December 2012.

[9] Yong Wang, Jianhua Ge, Linhua wang and Jing Li, "Reduction of PAPR of OFDM signals Using Nonlinear Companding Transform", Wireless Pers Commun, pp. 383-397, August 2012.

[10] Sandeep Bhad, Pankaj Gulhane, A.S. Hiwale "PAPR Reduction Scheme for OFDM" Procedia Technology, pp. 109 - 113, May 2012.

[11] Xiao Yue, Bai WenLing, Dan LiLin, WU Gang and LI ShaoQian, "Performance Analysis of Peak Cancellation in OFDM Systems", Science China Information Sciences, Vol. 55, pp. 789-794, April 2012.

[12] Hideki Ochiai and Hideki Imai, " Performance of the Deliberate Clipping with Adaptive Symbol Selection for Strictly Band-Limited OFDM Systems", IEEE journal on Selected Areas in Communication, Vol.18, pp. 2270-2277, November 2000.

[13] Hideki Ochiai and Hideki Imai, "Performance Analysis of Deliberately Clipped OFDM Signals", IEEE Transactions on Communications, Vol. 50, pp. 89-101, January 2002.

[14] E.S. Hassan, XU Zhu, S.E. El-Khamy, M.I. Dessouky, S.A. El-Dolil, F.E. Abd El-Samie, "Peak-to-average power ratio reduction using selective mapping with unequal power distribution", J. Cent. South Univ., pp. 1902-1908, March 2012.

[15] Stephane Y. Le Goff, Boon Kien Khoo, Charalampos C. Tsimenidis, and Bayan, S. Sharif "A Novel Selected mapping technique for PAPR reduction in OFDM systems", IEEE Transactions on Communications, VOL. 56, pp. 1775-1779, November 2008.

[16] Chunjiang Duanmu, Hongtao Chen "Reduction of the PAPR in OFDM Systems by Intelligently Applying Both PTS and SLM Algorithms", Wireless Pers Commun, July 2013. 